\title{
Resequencing of global Tartary buckwheat accessions reveals multiple domestication events and key loci associated with agronomic traits
}

Kaixuan Zhang ${ }^{1 \dagger}$, Ming He ${ }^{1 \dagger}$, Yu Fan ${ }^{1 \dagger}$, Hui Zhao ${ }^{1 \dagger}$, Bin Gao ${ }^{1 \dagger}$, Keli Yang ${ }^{1}$, Faliang Li $i^{2}$, Yu Tang ${ }^{1}$, Qiang Gao ${ }^{3}$, Tao Lin ${ }^{4}$, Muriel Quinet ${ }^{5}$, Dagmar Janovská ${ }^{6}$, Vladimir Meglič, Jacek Kwiatkowski ${ }^{8}$, Olga Romanova9 Nikhil Chrungoo $^{10}$, Tatsuro Suzuki ${ }^{11}$, Zlata Luthar ${ }^{12}$, Mateja Germ ${ }^{12}$, Sun-Hee Woo ${ }^{13}$, Milen I. Georgiev ${ }^{14,15}$ and Meiliang Zhou $^{\text {**t }^{*}}$ (D)

\author{
* Correspondence: zhoumeiliang@ \\ caas.cn \\ ${ }^{\dagger}$ Kaixuan Zhang, Ming He, Yu Fan, \\ Hui Zhao, Bin Gao and Meiliang \\ Zhou contributed equally to this \\ work. \\ ${ }^{1}$ Institute of Crop Sciences, Chinese \\ Academy of Agricultural Sciences, \\ Room 107, Ziyuan North Building, \\ Xueyuan South Road No. 80, \\ Haidian District, Beijing 100081, \\ China \\ Full list of author information is \\ available at the end of the article
}

\begin{abstract}
Background: Tartary buckwheat (Fagopyrum tataricum) is a nutritionally balanced and flavonoid-rich crop plant that has been in cultivation for 4000 years and is now grown globally. Despite its nutraceutical and agricultural value, the characterization of its genetics and its domestication history is limited.

Results: Here, we report a comprehensive database of Tartary buckwheat genomic variation based on whole-genome resequencing of 510 germplasms. Our analysis suggests that two independent domestication events occurred in southwestern and northern China, resulting in diverse characteristics of modern Tartary buckwheat varieties. Genome-wide association studies for important agricultural traits identify several candidate genes, including FtUFGT3 and FtAP2YT1 that significantly correlate with flavonoid accumulation and grain weight, respectively.
\end{abstract}

Conclusions: We describe the domestication history of Tartary buckwheat and provide a detailed resource of genomic variation to allow for genomic-assisted breeding in the improvement of elite cultivars.

Keywords: Buckwheat, Genomic variation, Domestication, GWAS

\section{Background}

Buckwheat provides balanced essential amino acids, resistance starch, vitamins, and minerals to human diets, and processes rich bioactive flavonoids, such as rutin, quercetin, (iso) vitexin, and epicatechin, with therapeutic effects on diabetes, hypertension, and hyperlipidemia $[1,2]$. This gluten-free crop has been traditionally used as a staple food for centuries in many high-altitude areas, such as southwestern China, Nepal, and Bhutan, and is an important raw material for functional food production. The

(c) The Author(s). 2021 Open Access This article is licensed under a Creative Commons Attribution 4.0 International License, which permits use, sharing, adaptation, distribution and reproduction in any medium or format, as long as you give appropriate credit to the original author(s) and the source, provide a link to the Creative Commons licence, and indicate if changes were made. The images or other third party material in this article are included in the article's Creative Commons licence, unless indicated otherwise in a credit line to the material. If material is not included in the article's Creative Commons licence and your intended use is not permitted by statutory regulation or exceeds the permitted use, you will need to obtain permission directly from the copyright holder. To view a copy of this licence, visit http://creativecommons.org/licenses/by/4.0/. The Creative Commons Public Domain Dedication waiver (http://creativecommons.org/publicdomain/zero/1.0/) applies to the data made available in this article, unless otherwise stated in a credit line to the data. 
cultivation of buckwheat can be traced back to about 4000 years ago [3], while it is still at the early stage of domestication and exhibits severe seed shattering and plant lodging. Buckwheat belongs to the Polygonaceae family and Fagopyrum genus that contains 21 species. The most widely cultivated species include common buckwheat (Fagopyrum esculentum) and Tartary buckwheat (Fagopyrum tataricum), which are grown on all continents as a result of their high ecological adaptability, short growth period, and tolerance of low-nutrient conditions. The improvement of this underutilized pseudocereal crop for ideal varieties with higher yield and nutrition could be harnessed in attempts to face the booming challenges of limited land and human nutritional needs.

Tartary buckwheat (F. tataricum) is homostylous and self-pollinated and has higher yield and flavonoids than the self-incompatible common buckwheat [4]. It is particularly rich in rutin that has therapeutic potential in Alzheimer's disease $[5,6]$. The cultivated Tartary buckwheat has been considered to originate and domesticate in the southwestern China based on the discovery and characterization of its wild relatives, literature evidence, and traditional catering culture [7-9]. The human selection and geographical isolation have resulted in the diversity of phenotypes and genotypes, providing abundant resources for Tartary buckwheat breeding. However, current cultivars of Tartary buckwheat are mainly selected individually from elite landraces, and the deficiency of genomic information and quantitative trait loci has until recently been the major limitation for modern breeding.

The first report of Fagopyrum genomics is a draft assembly of common buckwheat genome generated by next-generation sequencing (NGS), which was successfully used to identify novel candidate genes controlling heteromorphic self-incompatibility of common buckwheat [10]. Subsequently, a high-quality, chromosome-scale Tartary buckwheat genome sequence of $489.3 \mathrm{Mb}$ was released, and 33,336 high-confidence genes were annotated based on expression evidence [11]. In this study, a worldwide collection of Tartary buckwheat germplasm resources, along with the availability of Tartary buckwheat genome sequence and enhanced capacity of sequencing, makes it possible to construct a comprehensive database of the genome variation. Here, we report the population structure and multiple domestication events of Tartary buckwheat by resequencing the genomes of 510 global accessions of wild and cultivated genotypes. The genome-wide association study (GWAS) was performed to identify key loci and genes associated with several important agronomic traits.

\section{Results}

\section{Genome-wide variations and population structure}

We resequenced 510 worldwide Tartary buckwheat germplasm samples collected from 14 countries representing various geographical regions, including 483 landraces and 27 wild accessions, and 7 other Fagopyrum genus species (F. esculentum, F. leptopodum, F. qiangcai, F. pugense, F. rubifolium, F. gracilipedoides, F. caudatum) collected from Sichuan, China (Fig. 1a; Additional file 1: Figure S1; Additional file 2: Table S1). A total of $3.98 \mathrm{~Tb}$ of raw data were generated, with an average sequencing depth of $12.65 \times$ and 91.72\% genome coverage. We identified a final set of 1,095,748 single-nucleotide polymorphisms (SNPs) (Additional file 2: Table S2) and 116,516 indels (1-50 bp in length) (Additional file 1: Figure S2; Additional file 2: Table S3). Site frequency spectrum (SFS) 


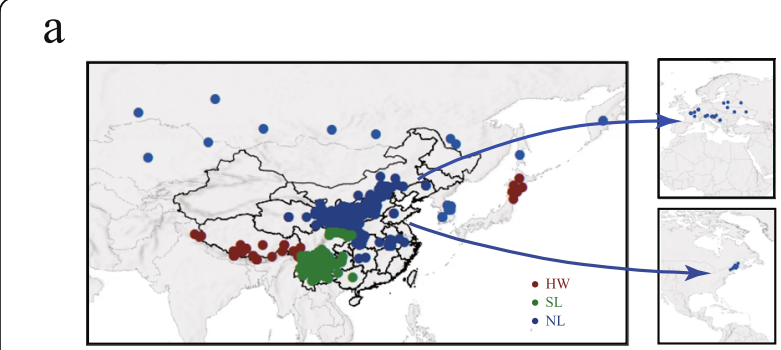

$\mathrm{b}$

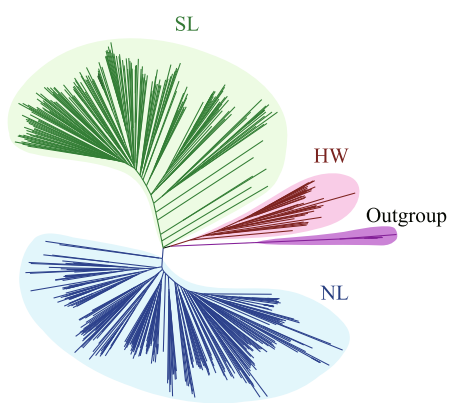

e

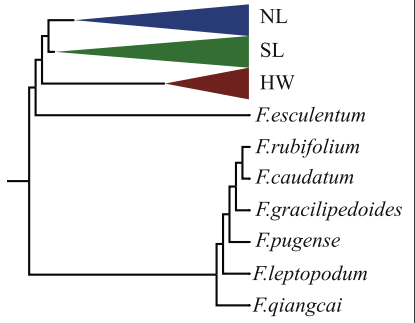

c

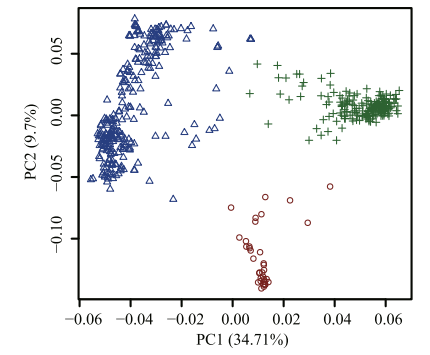

d

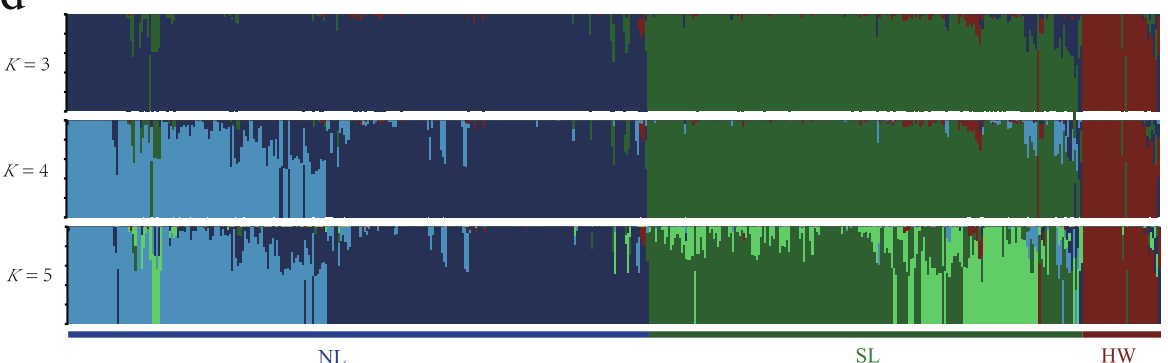

Fig. 1 Geographic distribution and population structure of resequenced accessions from Fagopyrum species. a Geographic distributions of Tartary buckwheat accessions. Each accession is represented by a dot on the map. The spread routes are shown with blue lines, which represent from northern China to other countries. HW, Himalayan Wild accession; SL, Southwest Landraces; NL, North Landraces. b Neighbor-joining tree of 517 germplasms, including 510 Tartary buckwheat accessions and 7 other Fagopyrum species. Branch colors indicate different groups: group HW (red), group SL (green), group NL (blue), and outgroup (purple), matching the colors shown in a. c Principal component analysis of Tartary buckwheat accessions, showing the first two components. Colors correspond to the phylogenetic tree grouping. $\mathbf{d}$ The population structure analysis with different numbers of clusters $(K=3,4$, and 5$)$ matches the phylogenetic tree. The $x$ axis lists the different accessions that are consistent with those in the phylogenetic tree. e Phylogenetic tree of the outgroup species

including folded and unfolded was applied to confirm the reasonableness of SNPs called from the whole-genome (Additional file 1: Figure S3). Most of the SNPs were located in intergenic regions and $2.3 \%$ were present in coding sequences, consisting of 8847 synonymous SNPs and 14,944 non-synonymous SNPs (Additional file 2: Table S2). This 1.69 ratio of non-synonymous to synonymous SNPs is higher than in pigeonpea (1.18) [12], chickpea (1.20) [13], castor (1.39) [14], and soybean (1.61) [15]. The Ts/ $\mathrm{Tv}$ (transition/transversion) ratio of 2.175 (Additional file 2: Table S2) for Tartary buckwheat is higher than that for black gram (1.58) [16], tomato (1.75) [17], and maize (1.02) [18]. Variants exhibit potential large effects composed of 814 damaging SNPs causing the gain/loss of stop codons and 764 indels resulting in a frameshift. SNPs were 
validated using the PCR-based sequencing strategy, and the accuracy was estimated to be $95.1 \%$, suggesting high reliability for SNP identification (Additional file 2: Table S4).

The phylogenetic analysis divided 510 Tartary buckwheat accessions into three major monophyletic clades (Fig. 1b; Additional file 1: Figure S4): group HW (Himalayan wild accessions) including 27 wild accessions and 9 landraces, group SL (Southwestern landraces) including 203 landraces, and group NL (Northern landraces) including 271 landraces. These results were further supported by principal component analysis (Fig. 1c) and model-based clustering analysis (Fig. 1d), which was as well consistent with DAPC analysis (Additional file 1: Figure S5). As expected, the phylogenetic tree suggested that Tartary buckwheat has a closer relationship to $F$. esculentum, the other cultivated buckwheat species, compared with other Fagopyrum genus species from the outgroup (Fig. 1e), which is consistent with the previous study [19].

The three groups displayed clearly different geographic distributions: wild accessions in HW mostly came from the Himalayan region; SL mainly included landraces from southwest China including Sichuan, Yunnan, and Guizhou provinces; and landraces from north and central China, Korea, Central Asia and Asian Russia, Europe, and North America were clustered in NL (Additional file 1: Figure S4; Additional file 2: Table S1). The genetic differentiation value $\left(F_{\mathrm{ST}}\right)$ for Chinese and abroad individuals in NL (0.045) was much lower than the value for HW and abroad accessions of NL (0.16) (Additional file 2: Table S5). The phylogenetic map and $F_{\mathrm{ST}}$ values suggest a possible migration route of Tartary buckwheat from the northern China rather than the origin place to the other countries. Interestingly, only 3 accessions from Japan were found in cultivated groups ( 2 in NL and 1 in SL), and the rest $(n=7)$ were all grouped together with wild accessions, suggesting a direct import from Himalayan region to Japan besides the introduction from Russia, which was also recorded by Suzuki et al. [20, 21].

Cultivated groups SL and NL displayed significant advantages compared with the wild group HW in terms of plant development, yield, and quality traits, resulting from the artificial selection of favorable phenotypic traits (Additional file 1: Figure S6 and S7; Additional file 2: Table S6). Pairwise genome-wide $F_{\mathrm{ST}}$ values for HW with SL (0.173) and HW with NL (0.193) (Additional file 2: Table S5) indicated the notable genetic divergences between the wild group and each cultivated group, which was shaped by domestication.

\section{Independent domestication and divergence between SL and NL}

To identify potential selective signals associated with Tartary buckwheat domestication, the cross-population composite likelihood ratio test (XP-CLR) was performed in the comparisons of HW versus SL and HW versus NL. Above the dashed horizontal thresholds of top 5\%, we identified 150 sweeps between HW and SL containing 3415 putative genes (Fig. 2a; Additional file 2: Table S7), and 156 sweeps between HW and NL containing 3006 putative genes (Fig. 2b; Additional file 2: Table S8), which covered $8.0 \%(39 \mathrm{Mb})$ and $8.5 \%(41 \mathrm{Mb})$ of the assembled genome, respectively. It was notable that only 19 sweeps $(4.1 \mathrm{Mb})$ and 420 genes overlapped for regions with selective signatures between SL and NL (Additional file 1: Figure S8), suggesting a likely independent domestication process driven by human intervention in the two genetically and geographically distinct groups. In addition to XP-CLR, de-correlated composite of multiple 


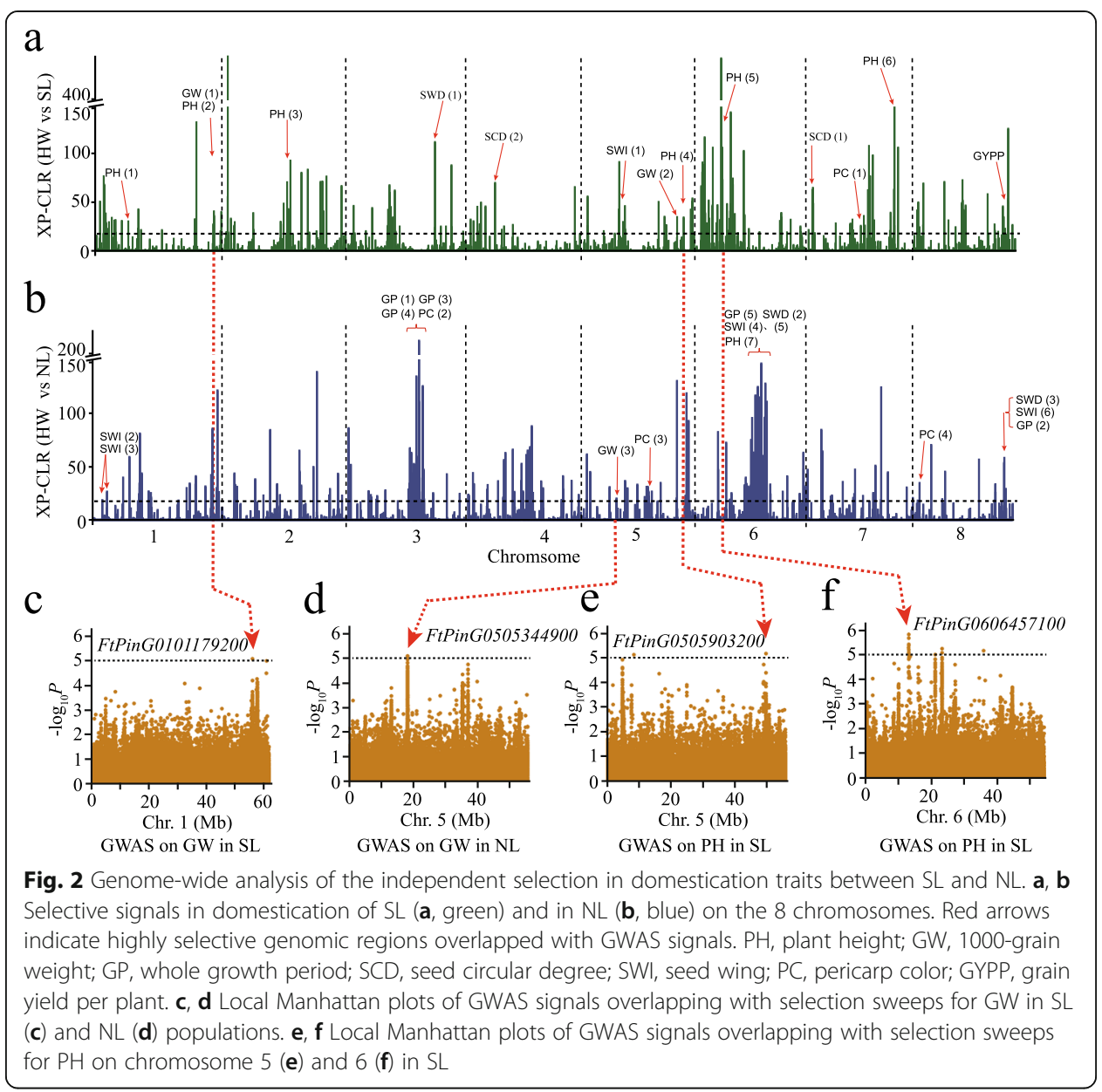

signals (DCMS) approach was also applied to detect selective sweeps. Above the dashed horizontal thresholds of top 10\%, 146 sweeps $(45 \mathrm{Mb})$ between HW and SL (Additional file 1: Figure S9a; Additional file 2: Table S9) and 112 sweeps ( $45 \mathrm{Mb}$ ) between HW and NL (Additional file 1: Figure S9b; Additional file 2: Table S10) were generated. Here, we identified $26.6 \mathrm{Mb}(59 \%)$ and $30.6 \mathrm{Mb}(68 \%)$ overlaps with sweeps identified by XP-CLR in SL and NL, respectively, indicating the reliability of two approaches.

To elucidate the genomic regions that underlie the remarkable phenotypical differences between SL and NL, we calculated the population differences and found 34 sweeps (top 5\% $F_{\mathrm{ST}}$, Additional file 1: Figure S10a; Additional file 2: Table S11). The results from $F_{\mathrm{ST}}$ and the comparison of genetic diversity $\left(\pi_{\text {wild }} / \pi_{\text {landrace }}\right)$ were combined to look for the unique selective sweep regions in SL and NL (Additional file 2: Table S12 and S13). We identified 4 unique selective sweeps in SL (Additional file 1: Figure S10b; Additional file 2: Table S14) and 8 in NL (Additional file 1: Figure S10c; Additional file 2: Table S14). The further exploitation and functional investigation of genes conferring genetic differentiation between SL and NL would contribute to the interpretation of Tartary buckwheat domestication process.

GWAS based on the Efficient Mixed-Model Association eXpedited (EMMAx) was performed for 10 traits to further determine the selected loci related to important agronomic traits during domestication (Additional file 1: Figure S9S11-S15S17). We found 
31 GWAS signals that overlapped with selective sweeps identified by XP-CLR for plant height $(\mathrm{PH}), 1000$-grain weight $(\mathrm{GW})$, whole growth period (GP), seed circular degree (SCD), seed wing (SWI), seed width (SWD), pericarp color (PC), and grain yield per plant (GYPP) (Fig. 2a, b; Additional file 2: Table S15). GWAS analysis generated 34 signals that overlapped with selective sweeps identified by DCMS for GW, GP, seed length (SDL), SWD, SCD, SWI, PC, and PH (Additional file 2: Table S16). Comparing with XP-CLR, we found 22 overlapped GWAS signals (9 in SL and 13 in NL) for GW, GP, SWD, SCD, SWI, PC, and PH (Additional file 2: Table S16). The alignment with GWAS analysis and $F_{\mathrm{ST}}$ (Additional file 1: Figure S18-S21) generated 8 signals that are associated with GW, GP, SWD, SWI, and PC and overlapped with the divergent regions (Additional file 1: Figure S10a; Additional file 2: Table S17).

In two distinct selective sweeps of SL and $\mathrm{NL}$ associated with 1000-grain weight, we found a gene FtPinG0101179200 on chromosome 1 (Fig. 2c), encoding the 13S globulin seed storage protein [22], and a gene FtPinG0505344900 on chromosome 5 (Fig. 2d), encoding an auxin-induced protein. For plant height, 6 significant selective sweeps were found in SL and only 1 in NL. By further analysis of candidate genes related to plant growth in these association regions, we identified a protein kinase gene (FtPinG0505903200) in a selective sweep of SL on chromosome 5 (Fig. 2e), and its Arabidopsis homolog LRR kinase AtVRLK1 was involved in switching between cell elongation and secondary cell wall thickening [23]. A translation factor gene related to plant height, FtPinG0606457100, was found on chromosome 6 in the selective sweep of SL as well (Fig. 2f), whose orthologous gene in Arabidopsis plays a crucial role in plant growth [24]. These genes were all confirmed via the factored spectrally transformed linear mixed models (FaST-LMM) (Additional file 1: Figure S22). These results supported that Tartary buckwheat underwent two independent domestication events, which was shaped by diverse genetic pathways in SL and NL, respectively.

\section{Genome-wide association with flavonoid metabolism}

The richness of flavonol compounds appears as one of the most prominent healthy and pharmaceutic properties of Tartary buckwheat. Here, we employed GWAS analysis by both FaST-LMM and EMMAx models for 480 accessions to identify potential genes that are prominently correlated with the contents of three flavonols (Additional file 1 : Figure S23-S25), including quercetin (QC), rutin (RC), and kaempferol-3-O-rutinoside (KC). One significant association with the kaempferol-3-O-rutinoside content was identified on chromosome 1 (Fig. 3a; Additional file 1: Figure S24; Additional file 2: Table $\mathrm{S} 18)$, and 20 candidate genes were found in this association region (4.52-4.72 Mb) (Fig. 3b, c; Additional file 2: Table S19). The peak SNP (Ft1:4617722, A/G) generated three haplotypes, Hap.1 (AA), Hap.2 (AG), and Hap.3 (GG), and located at the promoter of FtUFGT3 (FtPinG0100123400) gene (Fig. 3d), encoding an UDPglucosyltransferase that can catalyze anthocyanin to anthocyanin-3-O-glucoside [25]. The expression of FtUFGT3 in seeds was remarkably higher than the other candidate genes and decreased during the seed maturation from 13 to 25 DPA (days postanthesis) (Fig. 3e). We further found that the Hap.1 correlated with higher kaempferol-3-O-rutinoside content and that the Hap.3 correlated with lower content (Fig. 3f). The subsequent test of the FtUFGT3 gene expression showed obviously the positive correlation 


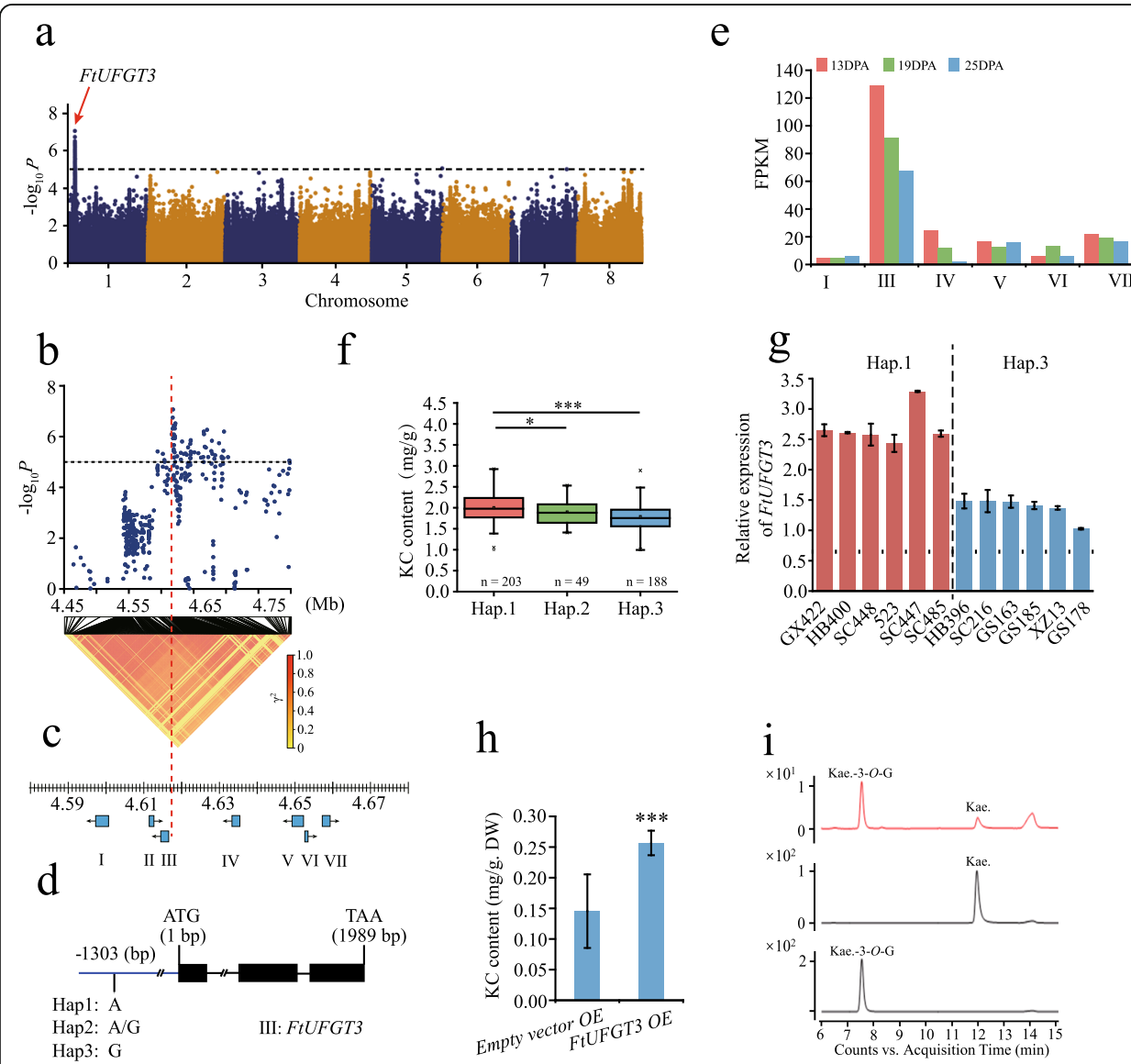

Fig. 3 Identification of the FtUFGT3 gene associated with kaempferol-3-O-rutinoside content. a Manhattan plot for GWAS on kaempferol-3-O-rutinoside content (KC) in the full population. The dashed line indicates the threshold $-\log P=5$. The red arrow indicates the SNP in FtUFGT3. $\mathbf{b}$ Local Manhattan plot (top) and linkage disequilibrium heat map (bottom) for the gene associated with KC. c Schematic representation of genes in the association region. I, FtPinG0100123000; II, FtPinG0100123100; III, FtUFGT3; IV, FtPinG0100123600; V, FtPinG0100123800; VI, FtPinG0100123900; VII, FtPinG0100124100. d Schematic representation of the FtUFGT3 genomic sequence. Exons and introns are represented by boxes and lines, respectively. The blue line represents the promoter and 3' UTR. The causal SNP locates in the promoter - $1303 \mathrm{bp}$ that is marked by a black line. Hap1, haplotype 1; Hap2, haplotype 2; Hap3, haplotype 3. e Expression of genes from c during seed development excluding II. DPA, days postanthesis. f Box plots show KC in three haplotypes (Hap.). ${ }^{*} P<0.05,{ }^{* *} P<0.001$, Student's $t$ test. $\mathbf{g}$ Relative expression levels of FtUFGT3 in different buckwheat accessions of Hap.1 and Hap.3. Data are mean \pm SD. $\mathbf{h}$ KC in Tartary buckwheat transgenic hairy roots overexpressing FtUFGT3, mixed by five lines. Data are mean \pm SD. ${ }^{* * *} P<0.001$, Student's $t$ test. i Enzyme assay of FtUFGT3 in vitro. Up for reaction product of MBP-FtUFGT3 protein and kaempferol standard; middle for kaempferol standard; below for kaempferol-3-O-glucoside standard. Kae., kaempferol

with kaempferol-3-O-rutinoside accumulation (Fig. 3g). Overexpression of FtUFGT3 in Tartary buckwheat hairy roots achieved elevated content of kaempferol-3-O-rutinoside in vivo (Fig. 3h; Additional file 1: Figure S26), and in vitro enzyme assays showed that FtUFGT3 catalyzed kaempferol into kaempferol-3-O-glucoside (Fig. 3h; Additional file 1: Figure S27). These results demonstrated that FtUFGT3 was involved in flavonoid metabolism.

For the quercetin content (QC), we found a candidate gene, FtPinG0100487500 (Additional file 1: Figure S25; Additional file 2: Table S20), encoding a glutathione transferase, whose orthologs in Arabidopsis (TT19) [26], tea (CsGSTa, b, c) [27], and peach (PpGST1) [28] have been reported to be essential for the storage of anthocyanins, 
flavonols, and proanthocyanidins. Pericarp color (PC) shaped by the accumulation and oxidation of flavonoids is also one of the key quality traits during artificial selection for different use and preference. We identified a significant GWAS signal on chromosome 2 using the black and non-black accessions (Additional file 1: Figure S28a), and the peak SNP (Ft2:44531080 C/G) yielded three haplotypes, Hap.1 (GG), Hap.2 (CG), and Hap.3 (CC). More than 90\% of Hap.1 gathered in the black seeds and 99\% of Hap.3 exhibited non-black type (Additional file 1: Figure S28b), indicating the significant correlation between Hap.1 and black pericarp color. Among the 9 genes located in this association region (44.43-44.63 Mb), the FtPinG0202040000 gene (Additional file 1: Figure S28c; Additional file 2: Table S20) is homologous to Arabidopsis TT2 that encodes a MYB transcription factor well-known as the key positive regulator of proanthocyanidin accumulation in developing seed [29]. In summary, all these genetic loci and candidate genes can be potentially used to improve the quality of buckwheat.

\section{Genome-wide association with agronomic traits}

The agronomic characters, such as yield and growth period, always come to the first in crop breeding, of which the regulatory mechanism is complicated. Using GWAS (FaST-LMM and EMMAx models) for 1000-grain weight that quantifies the yield directly (Additional file 1: Figure S29), we identified 2 non-synonymous SNPs that were located at FtPinG0404616900 (C/G, Ft4:46350596) and FtPinG0280000714 (T/G, Ft2: 37407976), which encode an AP2 transcription factor and an unknown protein, respectively (Additional file 2: Table S21 and S22). Therefore, FtPinG0404616900 (FtAP2YT1) was chosen for further functional investigation as the candidate gene associated with the 1000-grain weight trait (Fig. 4a). We identified 2 haplotypes, Hap.1 (CC) correlating with lower grain weight and Hap.2 (CG) correlating with higher 1000-grain weight (Fig. 4b, c). This non-synonymous SNP (C/G) led to an amino acid change from Pro to Ala at the position of the second $\beta$-sheet, where it is close to its DNA binding domain [30] (Fig. 4d). Yeast one-hybrid assay showed a prominently increased binding activity of FtAP2YT1 ${ }^{\text {Ala }}$ to the GCC cis-element compared to FtAP2YT1 ${ }^{\text {Pro }}$ (Fig. 4e; Additional file 1: Figure S30). We further selected 7 potential target genes of FtAP2YT1 with the GCC cis-element that correlated with grain weight (Additional file 2: Table S23). The expression of three genes, FtPinG0505979100, FtPinG0707412500, and FtPinG0708109000, was significantly higher in accessions of higher grain weight carrying Hap.2 than that of lower grain weight carrying Hap.1 (Fig. 4f-h). These results indicate that FtAP2YT1 may play an important role in regulating the expression of genes involved in 1000-grain weight.

Seed size is also an important factor that determines crop yield. Our GWAS analysis identified 2 candidate genes associated with seed width, including the above gene (FtPinG0404616900) related to 1000-grain weight (Additional file 1: Figure S31; Additional file 2: Table S20). FtPinG0100980400 encodes a tryptophan aminotransferaserelated protein that has been reported to be critical for grain yield via auxin biosynthesis in rice [31] and wheat [32]. Candidate genes in the important agronomic traits associated regions can be potentially used for the yield improvement of Tartary buckwheat cultivars. 

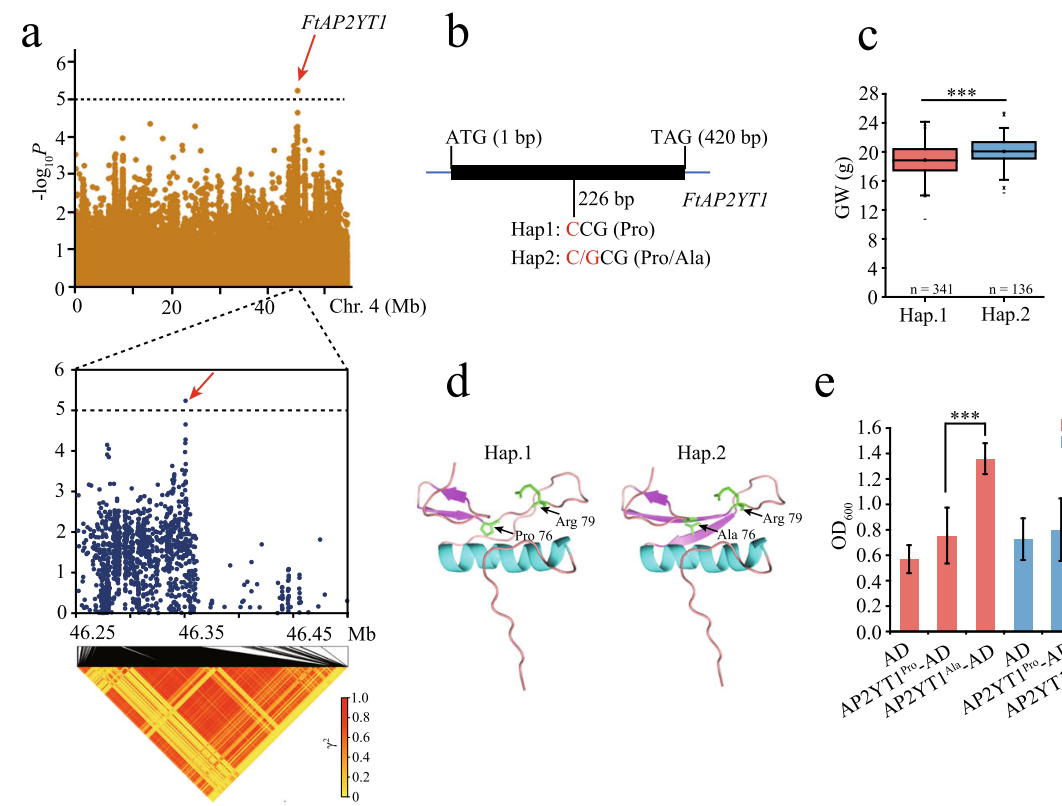

e
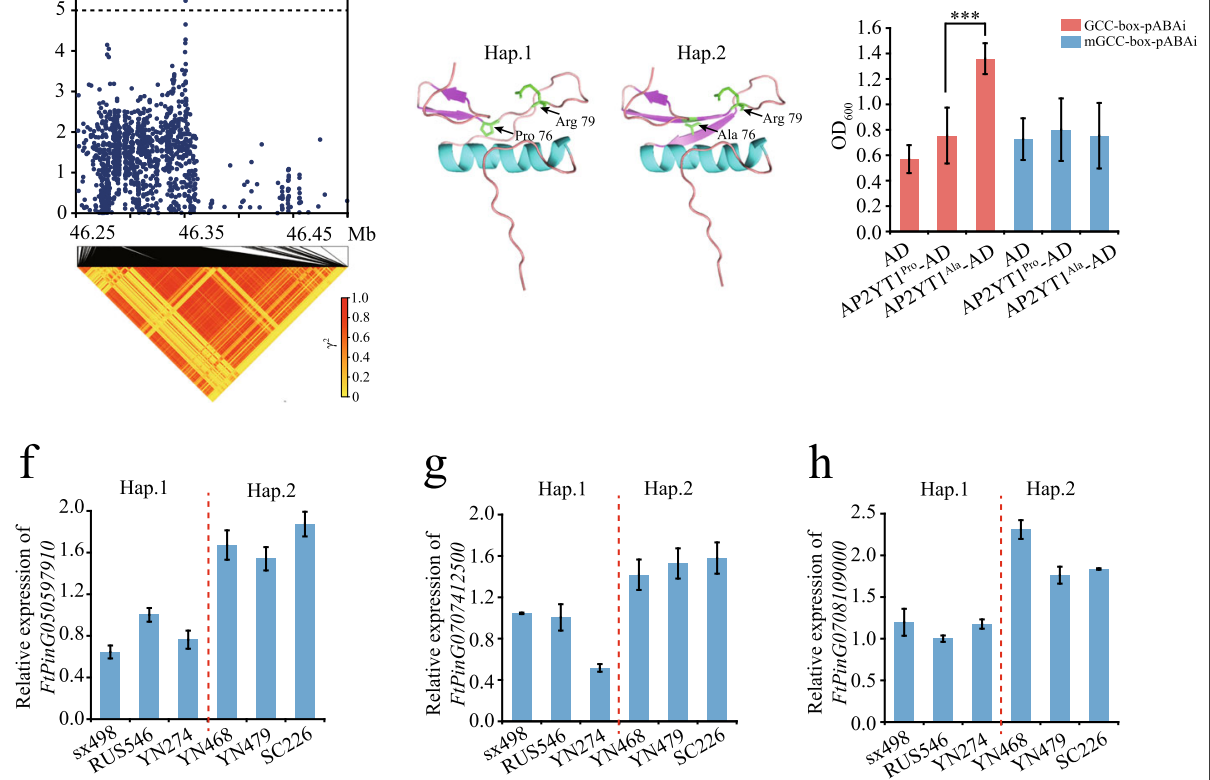

Fig. 4 Identification of the candidate gene FtAP2YT1 associated with 1000-grain weight. a Manhattan plot for GWAS on 1000-grain weight (GW). The dashed line indicates the threshold $-\log P=5$. The red arrow indicates the SNP in FtAP2YT1. b Structure of FtAP2YT1 genomic sequence containing only one exon that is represented by a box. The non-synonymous SNP at 226 bp is marked by a black line. Hap1, haplotype 1; Hap2, haplotype 2. c Box plots show GW in two haplotypes (Hap.). ${ }^{* *} P<0.001$, Student's $t$ test. d Protein structure model of FtAP2YT1. Compared with site Pro76, Ala76 enlarged the $\beta$-sheet length, which functions in binding to the DNA. e Y1H analysis of the activity of FtAP2YT1 Pro and FtAP2YT1 ${ }^{\text {Ala }}$ binding to GCC-box and mGCC-box. Data are mean $\pm S D$. ${ }^{* * *} P<0.001$, Student's $t$ test. $\mathbf{f}-\mathbf{h}$ Expression of genes containing GCC-box that are potential targets of FtAP2YT1 in different accessions carrying Hap.1 and Hap.2, respectively

\section{Discussion}

The rich genetic diversity of Fagopyrum resources and the characterization of cultivated buckwheat ancestors in Himalayan region suggest the possible origin of cultivated buckwheat. The diffusion of buckwheat was then proposed that it was brought from China and southern Himalaya to the world after the birth of cultivated buckwheat $[3,33]$. In our research, the closely phylogenetic relationship between accessions from northern China and from outside China (Additional file 1: Figure S4), along with the $F_{\mathrm{ST}}$ value (Additional file 2: Table S5), provides evidences for the hypothesis that Tartary buckwheat was introduced from the northern China to the other countries after wild Tartary buckwheat was domesticated in China. Given the lack of archeological and palynological evidences and small number of wild accessions, this result has limited capacity to unravel the historical migration of Tartary buckwheat.

After the cultivation and artificial intervention for thousands of years, the 1000-grain weight increased and the whole growth period decreased significantly in cultivated Tartary 
buckwheat accessions comparing with wild accessions. However, unlike most gramineous and legume crops, this pseudocereal still possesses wild characterizations, including seed shattering, thick shell, tall stalk, plant lodging, and low yield. Besides the phenotypes, wellcharacterized domestication genes, such as sh4 for seed shattering of rice [34], tgal for naked grains of maize [35], PROG1 for tiller angle of rice [36], and $G$ for seed dormancy in soybean, rice, and tomato [37], were not found in the genetically selective sweeps obtained by multiple methods, supporting that the domestication process is still at the very early stage.

SL and NL landraces showed a clear geographic and genetic division, besides the phenotypic differences between the two groups, including seed size, plant height, whole growth period, and grain weight. The divergence between SL and NL landraces can be explained by the artificial selection for locally adapted and preferred traits. Those unique selective sweep regions and their underlying genes in SL and NL generated from the independent domestication process (Fig. 2; Additional file 1: Figure S18; Additional file 2: Table S15 and S16) provide potential valuable resources for molecular breeding of Tartary buckwheat. For instance, a protein kinase gene FtPinG0505903200 associated with plant height was identified in a selective sweep of SL (Fig. 2). This gene belongs to the leucine-rich repeat receptor-like protein kinase (LRR-RLK) family that is the largest group of receptor-like kinases in plants and plays crucial roles in development and stress responses [38]. Therefore, Tartary buckwheat LRR-RLKs were extracted and their conserved kinase domains were aligned with Arabidopsis LRR-RLKs. In total, the 199 buckwheat LRRRLKs were divided into 19 different subfamilies and the FtPinG0505903200 was clustered in the VIII-1 group (Additional file 1: Figure S32), which can provide insights into possible gene functions and mechanisms of functional divergence.

\section{Conclusion}

In the present study, from the abundant genome variations of Tartary buckwheat accessions including wild samples and landraces, we achieved genomic regions that have experienced selective sweeps corresponding to separate domestication events of SL and NL. Unique selective sweeps and candidate genes in the two geographically different groups might be useful for modern molecular breeding. In addition, using GWAS, we identified several genomic loci that contribute to the formation of important quality and yield traits of Tartary buckwheat. Functional analysis of a key enzyme gene FtUFGT3 associated with flavonoid metabolism and a transcription factor FtAP2YT1 associated with grain weight displayed their potential use on the improvement of elite Tartary buckwheat cultivars. All these loci generated by GWAS analysis conferred promising candidates for not only buckwheat but also other crop improvement.

In summary, this research provides extensive genomic resources to gain insights into the buckwheat origin, spread, and domestication and will promote future breeding in character improvement, especially in yield and quality.

\section{Methods}

\section{Plant materials and phenotyping}

For genome resequencing, we used 510 Tartary buckwheat accessions composed of 32 wild accessions, 478 landraces, and 7 other Fagopyrum genus species (F. esculentum, $F$. leptopodum, F. qiangcai, F. pugense, F. rubifolium, F. gracilipedoides, F. caudatum) as 
the outgroup, which were obtained from holdings of the National Crop Genebank of China (NCGC) and collections of worldwide buckwheat research groups. These accessions are mainly from 20 provinces in China as well as from South Korea, Japan, Russia, Poland, USA, Nepal, Bhutan, India, Slovenia, France, and Belgium, covering most areas where Tartary buckwheat is currently cultivated (Additional file 2: Table S1).

For phenotyping, a total of 480 Tartary buckwheat accessions were grown in Liangshan (Sichuan province, $27^{\circ} 59^{\prime} \mathrm{N}, 102^{\circ} 50^{\prime} \mathrm{E}$ ) and Zhaotong (Yunnan province, $28^{\circ}$ $36^{\prime} \mathrm{N}, 103^{\circ} 49^{\prime} \mathrm{E}$ ) in 2017 and 2018. All seeds were sown by hand in three replications on 12 April, and seeds were harvested in the middle of July. Three individual plants from each accession in each replication were used for measurements of plant height $(\mathrm{PH})$. The whole growth period (GP) was defined as the growing days from sowing to maturity. Mature seeds were harvested from the selected six plants for measurements of 1000-grain weight (GW), grain yield per plant (GYPP), seed length (SLE), seed width, (SWD) seed circular degree (SCD), seed wing (SWI), pericarp color (PC), and seed diameter (SD).

\section{Measurement of flavonoid content}

Seeds were smashed and filtered by the 40-mesh sieve after they were pre-dried at $105^{\circ} \mathrm{C}$ for $30 \mathrm{~min}$ and kept at $65^{\circ} \mathrm{C}$ to a constant weight. Samples $(0.2 \mathrm{~g})$ were ultrasonically extracted in $20 \mathrm{ml} 80 \%$ methanol at $50^{\circ} \mathrm{C}$ and $40 \mathrm{k} \mathrm{Hz}$ for $25 \mathrm{~min}$. The solution was then filtered through the $0.22-\mu \mathrm{m}$ organic microporous filter and analyzed by highperformance liquid chromatograph (HPLC, Agilent G6500 Series HPLC-QTOF). A RP18 column $(2.1 \mathrm{~mm} \times 75 \mathrm{~mm} \times 2.7 \mu \mathrm{m})$ was operated at $40^{\circ} \mathrm{C}$. The mobile phase consisted of a mixture of (A) water/formic acid (99.9/0.1, v/v) and (B) methanol/formic acid $(99.9 / 0.1, \mathrm{v} / \mathrm{v})$. The gradient program was set as follows: 0-13 min, 20\% (B); $13-$ 13.5 min, gradually rose to $50 \%$ (B); $13.5-17$ min, gradually decrease to $20 \%$ (B); $17-18$ min, keep 20\% (B); and $18.1 \mathrm{~min}$, stop. The contents of rutin (RC), quercetin (QC), and kaempferol-3-O-rutinoside $(\mathrm{KC})$ were calculated by comparing the HPLC peak area with authenticated standards (Sigma-Aldrich, USA). Three replicates were performed for every sample.

\section{DNA extraction and sequencing}

Genomic DNA was extracted from young leaves using the cetyltrimethylammonium bromide (CTAB) method. At least $1 \mu \mathrm{g}$ genomic DNA for each accession was used to construct a sequencing library according to vendor-provided instructions (Illumina). Paired-end sequencing libraries with an insert size of approximately $350 \mathrm{bp}$ were sequenced on an Illumina NovaSeq 6000 platform in Berry Genomics. Trimmomatic v0.33 was used to trim the Illumina fastq and remove adapters based on the manufacturer's adapter sequences. Raw data of fastq format were then processed through inhouse perl scripts. In this step, clean data were obtained by removing reads containing adapter, reads containing poly- $\mathrm{N}$, and low-quality reads from raw data.

\section{Read alignment and variant calling}

All the sequenced reads for each accession were mapped to the assembly genome [11] (http://www.mbkbase.org/Pinku1/) using the Burrows-Wheeler Aligner program [39] 
(BWA 0.7.5a) with default parameters. We sorted the alignments according to mapping coordinates in samtools $[40,41](0.1 .19)$. After removing reads with low mapping quality $(\mathrm{MQ}<30)$, both paired-end and single-end mapped reads were used for SNP detection across the entire sample set of buckwheat accessions using the GATK toolkit [42] (version 3.4-46-gbc02625). Reads having a mean of approximately $12 \times$ depth for each individual and $>70 \%$ mapping rate of the buckwheat genome were retained for SNP calling. SNPs and small indels ( $1-50 \mathrm{bp}$ ) were called using the GATK UnifiedGenotyper module for diploids with -stand_call_conf 50-stand_emit_conf 10-dcov 1000 to call variants. We filtered variants both per population and per individual using GATK according to stringent filtering criteria. For SNPs of population filter: (a) QUAL > 30.0; (b) $\mathrm{QD}>5.0$; (c) $\mathrm{FS}<60.0$; (d) $\mathrm{MQ} 0 \geq 4 \& \&\left(\left(\mathrm{MQ0} /\left(1.0^{*} \mathrm{DP}\right)\right)>0.1\right)$; (e) $\mathrm{DP}>5$. We are working on a non-model organism and there is no SNP data available, so following GATK best practice tutorial, we chose Hard Filtering instead of Variant Recalibration (VQSR) method to filter our variants callset (https://gatk.broadinstitute.org/hc/en-us/ articles/360036434492-VariantFiltration). According to the GATK toolkit, if there were more than 3 SNPs clustered in a 10-bp window, all three SNPs were considered as false positives and removed according to the GATK toolkit. All SNPs and indels were assigned to specific genomic regions and genes using ANNOVAR [43] based on buckwheat genome annotations. To make sure that SNPs called from the whole-genome resequencing data are reasonable, site frequency spectrum (SFS) was applied [44] with the callset at population level based on MAF $>0.05$ and missing rate $<0.1$.

\section{SNP validation}

A total of 510 SNPs were selected randomly and detected by PCR-based sequencing in 10 Tartary buckwheat accessions, using PCR-based sequencing in more than 3 replicates (Additional file 2: Table S4). We aligned all the PCR products against the reference genome with DNAMAN software.

\section{Phylogenetic and population structure analyses}

We removed all SNPs with a minor allele frequency $\leq 0.05$ and a missing rate $>10 \%$ in all accessions. A subset of 1,094,031 SNPs were used for phylogenetic and population structure analysis. Vcf files were converted to hapmap format with custom perl scripts and to PLINK format file by PLINK v1.90 (http://pngu.mgh.harvard.edu/purcell/plink/). Under the p-distances model with bootstrapping (100), a neighbor-joining tree of all samples was constructed with TreeBest 1.9.2 [45]. SNPRelate [46] (1.18.1) was used to carry out principal component analysis (PCA), first by generating the genetic relationship matrix from which the first 3 eigenvectors were extracted. fastStructure [47] (version 1.0) was used for inferring population structure from large SNP genotype data sets. $K$ values were set from $K=3$ to $K=5$. Each $K$ value, as a putative number of populations set from 1 to 10 , was obtained with five independent runs with different starting seeds. The length of the burn-in period and number of MCMC replications after burnin were set to 50,000 and 100,000, respectively. The optimum number of subgroups $(K)$ was determined based on the $\log$ probability of the data $(\ln \mathrm{P}(K))$ and an ad hoc statistic $\Delta K$ method. To further confirm the result of the structure, the discriminant analysis of 
principal components (DAPC) was used to cluster genotypes independently of a priori haplotype designation using the R package adegenet v. 1.4.2 [48].

\section{Identification of selective sweeps}

To detect selective sweeps, the cross-population composite likelihood ratio test XPCLR v1.0 [49] was performed. We compared HR with SL and HR with NL group in 200-kb sliding windows with a step size of $100 \mathrm{~kb}$. The highest XP-CLR values, accounting for $5 \%$ of the genome, were considered as selected regions. Adjacent windows with high XP-CLR were grouped into a single region to represent the effect of a single selective sweep. In addition to XP-CLR, de-correlated composite of multiple signals (DCMS) approach [50] was performed and the comparison of genetic diversity ( $\pi_{\text {wild }} /$

$\pi_{\text {landrace }}$ ) was calculated to identify selective sweeps using the same 200-100-kb sliding window.

\section{Identification of genomic differentiation}

The level of genetic differentiation $\left(F_{\mathrm{ST}}\right)$ between populations was calculated in 200-kb intervals using PopGenome [51]. To detect differentiated regions, the average $F_{\mathrm{ST}}$ of all sliding windows across group II and NM were compared.

\section{GWAS and identification of the candidate genes}

Only SNPs with MAF $\geq 0.05$ and missing rate $\leq 0.1$ were used for GWAS, which resulted in $844,290,885,276$, and 1,094,031 SNPs for SL, NL, and the entire population (SL, NL, and HW), respectively. The association analysis was done with the Efficient Mixed-Model Association eXpedited program (EMMAx) [52] and the factored spectrally transformed linear mixed models (FaST-LMM) [53]. The effective number of independent SNPs was estimated as 988,845 , and thus, the significance threshold was estimated approximately $P=10^{-6}$.

According to the associated loci determined by GWAS, SNP types and locations were identified using the reference genome [11]. The total genes in each candidate region were analyzed and annotated by homologous comparison with Arabidopsis to narrow down the candidate genes.

\section{Enzyme assays}

The FtUGT3 CDS was inserted into the pMAL-C2X MBP [54] fusion expression vector and transformed into E. coli BL21. The MBP fusion proteins were extracted and immobilized onto amylose beads (New England Biolabs) with protein extraction buffer (20 $\mathrm{mM}$ Tris- $\mathrm{HCl}$ (pH 7.4), $0.2 \mathrm{M} \mathrm{NaCl}$, and $1 \mathrm{mM}$ EDTA). Protein was eluted using 20 $\mathrm{mM}$ Tris- $\mathrm{HCl}$ (pH 7.4), $0.2 \mathrm{M} \mathrm{NaCl}, 1 \mathrm{mM}$ EDTA, and $10 \mathrm{mM}$ maltose.

The reaction mixture ( $\mathrm{pH} 8.0,100 \mathrm{mM}$ Tris- $\mathrm{HCl}, 14 \mathrm{mM} \beta$-mercaptoethanol, $9 \mathrm{mM}$ UDP-glucose, and $100 \mu \mathrm{M}$ kaempferol) was added to $5 \mu \mathrm{g}$ purified protein and incubated at $37^{\circ} \mathrm{C}$ for $30 \mathrm{~min}$. The reaction was terminated by the freeze-dryer at $-40^{\circ} \mathrm{C}$. The dried reaction products were re-dissolved in $80 \%$ methanol, and $5 \mu \mathrm{l}$ of the solution was analyzed by LC-MS (Agilent G6500 Series HPLC-QTOF) to determine the product using the standards of kaempferol and kaempferol-3-O-glucoside (Sigma-Aldrich, USA). 


\section{Transgenic hairy roots}

The FtUGT3 CDS was inserted into the pCAMBIA 1307 vector and transformed into Agrobacterium A4 to generate transgenic hairy roots following previous methods [55-57]. Two weeks old sterile seedlings of Tartary buckwheat were cut and used as the explant for the infection with the Agrobacterium for $10 \mathrm{~min}$. After the co-culture on MS solid medium in the dark for $48 \mathrm{~h}$ at $25^{\circ} \mathrm{C}$, explants were washed by MS liquid medium containing $300 \mathrm{mg} / \mathrm{ml}$ cefotaxime and sterile water, and then cultured on MS solid medium containing $300 \mathrm{mg} / \mathrm{ml}$ cefotaxime in the growth chamber for the hairy root induction. The induced single hairy root lines were removed from explants after 2 weeks and put on MS solid medium with $100 \mathrm{mg} / \mathrm{ml}$ cefotaxime for detoxification and growth. The positively transgenic lines were tested by PCR and moved to MS liquid medium with $100 \mathrm{mg} / \mathrm{ml}$ cefotaxime shaking for 2 weeks in the dark at $22^{\circ} \mathrm{C}, 160 \mathrm{r} / \mathrm{min}$. Hairy roots were then harvested and dried for the measurement of kaempferol-3-O-rutinoside as described above.

\section{Yeast one hybrid $(\mathrm{Y} 1 \mathrm{H})$}

The cis-elements GCC-box (AGTGCCAAAAGCCGCCACACCCCT) and mGCC-box (AGTGCCAAAATCCACTACACCCCT) were inserted into pABAi vector as reporters, respectively. The reporters were linearized using restriction enzyme BbsI and transformed into $\mathrm{Y} 1 \mathrm{H}$ gold strain. FtAP2YT1 ${ }^{\text {Pro }}$ and $F t A P 2 Y T 1^{A l a}$ were inserted into pGADT7 vector containing a GAL4 transcriptional activation domain as effectors, respectively. The effectors were transformed into the $\mathrm{Y} 1 \mathrm{H}$ gold strain containing the reporter gene, respectively. Transformants were plated on minimal synthetic defined (SD)-glucose medium lacking Leu (-L) and selected on SD-L medium with Aureobasidin A. Y1H assay was performed according to the manufacturer's protocol (Matchmaker One-Hybrid System; Clontech; http://www.clontech.com/).

\section{Quantitative RT-PCR analyses}

Total RNA of 7-day-old buckwheat seedlings were extracted by RNApre Pure Plant Plus Kit (DP441, Tiangen, Beijing, China). Reverse transcription was carried out using the HiScript III RT SuperMix for qPCR (R323-01, Vazyme, Nanjing, China) according to the manufacturer's protocol. The qRT-PCR was performed as the protocol of ChamQ Universal SYBR qPCR Master Mix (Q711, Vazyme, Nanjing, China).

\section{Supplementary Information}

The online version contains supplementary material available at https://doi.org/10.1186/s13059-020-02217-7.

Additional file 1: Figure S1. Fagopyrum species used in this research. Figure S2. Distribution of small indels. Figure S3. Site frequency spectrum for all accessions. Figure S4. Neighbor-joining tree analysis of 517 buckwheat accessions (HW in red, SL in green, NL in blue) using SNPs detected in whole-genome resequencing data. Figure S5. Population structure of Tartary buckwheat with DAPC. Figure S6 and Figure S7. Quantification of agronomic and quality traits of 480 accessions in three groups. Figure S8. Venn diagram for the overlap of selective sweeps (a) and genes (b) correlated with independent domestication between SL and NL. Figure S9. Genome wide analysis of selection sweeps during independent domestication used by de-correlated composite of multiple signals (DCMS). Figure S10. Unique selective sweep regions analysis with FST and the comparison of nucleotide diversity. Figure S11. GWAS analysis of 1000-grain-weight in SL and NL, respectively. Figure S12. GWAS analysis of seed width in SL and NL, respectively. Figure S13. GWAS analysis of seed circular degree in SL and NL, respectively. Figure S14. GWAS analysis of whole growth period in $S L$ and NL, respectively. Figure S15. GWAS analysis of plant height in SL and NL, respectively. Figure S16 and Figure S17. GWAS analysis of agronomic traits in $S L$ and NL, respectively. Figure S18 to S21. GWAS analysis of differentiation traits. Figure S22. GWAS analysis of GW and PH in SL and NL used by FaST-LMM, respectively. Figure S23. GWAS analysis of rutin content. Figure S24. GWAS analysis of kaempferol-3-O-rutinoside content. Figure S25. GWAS analysis of quercetin content. Figure S26. Hair root transgenic system in buckwheat. Figure S27. In vitro enzyme assay of FtUFGT3. Figure S28. GWAS analysis of 
pericarp color. Figure S29. GWAS analysis of 1000-grain-weight. Figure S30. Y1H assay for the activity of FtAP2YT1 ${ }^{\text {ro }}$ and FtAP2YT1 ${ }^{\text {Ala }}$ binding to GCC-box and mGCC-box. Figure S31. GWAS analysis of seed width. Figure S32. Maximum likelihood tree of LRR-RLK genes.

Additional file 2: Table S1. Summary of all accessions sequenced in this study. Table S2. Distribution of SNPs within various genomic regions in Tartary buckwheat. Table S3. Distribution of indels within various genomic regions. Table S4. The validation of random selected SNPs by PCR methods. Table S5. Levels of genetic differentiation in different chromosomes. Table S6. 13 agronomic and quality traits used in GWAS. Table S7. Putative regions and genes experiencing domestication sweep between HW and SL. Table S8. Putative regions and genes experiencing domestication sweep between HW and NL. Table S9. Putative regions and genes experiencing domestication sweep between HW and SL calculated by DCMS. Table S10. Putative regions and genes experiencing domestication sweep between HW and NL calculated by DCMS. Table S11. Putative regions and genes experiencing differentiation sweep between SL and NL. Table S12. Putative regions and genes experiencing domestication sweep between HW and SL. Table S13. Putative regions and genes experiencing domestication sweep between HW and NL. Table S14. Regions identified by Fst approaches overlapped with the SL or NL unique selective sweep regions. Table S15. Overlaps between domesticated regions and GWAS signals for all traits. Table S16. Overlaps between domesticated regions and GWAS signals for all traits calculated by DCMS. Table S17. Overlaps between differentiated regions and GWAS signals for all traits. Table S18. SNP analysis associated with Ft1:4617722 for kaempferol-3-O-rutinoside content $(-\log P>5)$. Table S19. Identification of candidate genes associated with Ft1:4617722 on Chr.1 for kaempferol-3-O-rutinoside content. Table S20. Information of the candidate genes from GWAS analysis. Table S21. Total SNPs for GWAS on 1000-gain-weight $(-\log P$ >

5). Table S22. Identification of candidate genes associated with Ft4:46350596 on Chr.4 for 1000-gain-weight. Table S23. The information of FtPinG0404616900 potential target genes from GWAS on 1000-gain-weight. Table S24. Primers used in this study.

Additional file 3. Review history.

\section{Acknowledgements}

We thank Junzhen Wang and Ayi Shen for their efforts on the seed harvest and phenotyping. We thank Mengqi Ding, Cheng Cheng, Tianbin Tan, Cheng Chen, Guoying Gao, Xiaofang Wu, and Xiaoling Lu for their efforts on the preparation of plant samples and molecular experiments. We thank Ivan Kreft, Oksana Sytar, Christian Zewen, Grażyna Podolska, Galina Suvorova, Jianping Cheng, Jingjun Ruan, Chaoxia Sun, and the National Crop Genebank of China for providing most of the Tartary buckwheat germplasm resources.

\section{Review history}

The review history is available as Additional file 3.

\section{Peer review information}

Tim Sands was the primary editor of this article and managed its editorial process and peer review in collaboration with the rest of the editorial team.

\section{Authors' contributions}

K.Y., Y.T., M.Q., D.J., J.K., O.R., N.C., T.S., Z.L., M.G., S.H. W., and M.Z. contributed to the genetic material. K.Z., M.H., and B.G. contributed to the generation of whole-genome resequencing data. F.L., Y.F., and H.Z. performed the phenotyping and quality trait measurement. K.Z., M.H., B.G., Q.G., and T.L. performed the data analysis and/or figure design. Y.F. and H.Z. extracted the DNA and RNA, and performed the gene functional analysis. M.Z., V.M., and M.I.G. designed and managed the project. K.Z., M.H., and M.Z. wrote and finalized the manuscript, with the advice from Q.G. and T.L. All authors read and approved the paper.

\section{Funding}

This research was supported by the National Key R\&D Program of China (2018YFD1000705/2018YFD1000700,

2017YFE0117600), National Natural Science Foundation of China (31871536, 31801427, 31901511, 31911540469), European Union Horizon 2020 project ECOBREED (771367) and Planta SYST (SGA-CSA No. 739582 under FPANo. 664620), and Young Talent Supporting Plan of The Crop Science Society of China.

\section{Availability of data and materials}

All genomic sequence raw data sets for genetic diversity analysis and GWAS are available from NCBI under BioProject accession no. PRJNA600676 [58]. The scripts, codes, and analytic tools are deposited at the GitHub repository (https:// github.com/rahello/tartary_population) [59] and Zenodo with the access code DOl: https://doi.org/10.5281/zenodo. 3972746 [60].

\section{Ethics approval and consent to participate}

Not applicable.

\section{Competing interests}

The authors declare no competing interest.

\section{Author details}

${ }^{1}$ Institute of Crop Sciences, Chinese Academy of Agricultural Sciences, Room 107, Ziyuan North Building, Xueyuan South Road No. 80, Haidian District, Beijing 100081, China. ${ }^{2}$ Research Station of Alpine Crop, Xichang Institute of Agricultural Sciences, Liangshan 616150, Sichuan, China. ${ }^{3}$ BGI Genomics, BGI-Shenzhen, Shenzhen 58083, Guangdong, China. ${ }^{4}$ College of Horticulture, China Agricultural University, Beijing 100083, China. ${ }^{5}$ Groupe de Recherche en 
Physiologie Végétale (GRPV), Earth and Life Institute-Agronomy (ELI-A), Université catholique de Louvain, Croix du Sud 45, boîte L7.07.13, B-1348 Louvain-la-Neuve, Belgium. ${ }^{6}$ Gene Bank, Crop Research Institute, Drnovská 507, Prague 6, Czech Republic. ${ }^{7}$ Agricultural Institute of Slovenia, Hacquetova ulica, Ljubljana, Slovenia. ${ }^{8}$ Department of Plant Breeding and Seed Production, University of Warmia and Mazury in Olsztyn, Plac Łódzki 3, 10-724 Olsztyn, Poland. ${ }^{9}$ N.I. Vavilov All-Russian Institute of Plant Genetic Resources (VIR), Bol'shaya Morskaya, 42-44, St. Petersburg, Russia 190000. ${ }^{10}$ Department of Botany, North Eastern Hill University, Shillong 793022, India. ${ }^{11}$ Kyushu Okinawa Agricultural Research Center, National Agriculture and Food Research Organization, Suya 2421, Koshi, Kumamoto 861-1192, Japan. ${ }^{12}$ Biotechnical Faculty, University of Ljubljana, Jamnikarjeva 101, SI-1000 Ljubljana, Slovenia. ${ }^{13}$ Department of Crop Science, Chungbuk National University, Cheong-ju, Republic of Korea. ${ }^{14}$ Group of Plant Cell Biotechnology and Metabolomics, The Stephan Angeloff Institute of Microbiology, Bulgarian Academy of Sciences, Plovdiv, Bulgaria. ${ }^{15}$ Center of Plant Systems Biology and Biotechnology, Plovdiv, Bulgaria.

\section{Received: 15 February 2020 Accepted: 3 December 2020}

Published online: 12 January 2021

\section{References}

1. Joshi DC, Zhang K, Wang C, Chandora R, Khurshid M, Li J, et al. Strategic enhancement of genetic gain for nutraceutical development in buckwheat: a genomics-driven perspective. Biotechnol Adv 2020;39:107479.

2. Joshi DC, Chaudhari GV, Sood S, Kant L, Pattanayak A, Zhang K, et al. Revisiting the versatile buckwheat: reinvigorating genetic gains through integrated breeding and genomics approach. Planta. 2019;250:783-801.

3. Hunt HV, Shang X, Jones MK. Buckwheat: a crop from outside the major Chinese domestication centres? A review of the archaeobotanical, palynological and genetic evidence. Veget Hist Archaeobot. 2018;27:493-506.

4. Bonafaccia G, Fabjan N. Nutritional comparison of tartary buckwheat with common buckwheat and minor cereals. Zb Bioteh Fak Univ Ljublj Kmet. 2003;81:349-55.

5. Xu P, Wang S, Yu X, Su Y, Wang T, Zhou W, et al. Rutin improves spatial memory in Alzheimer's disease transgenic mice by reducing $A \beta$ oligomer level and attenuating oxidative stress and neuroinflammation. Behav Brain Res. 2014;264:173-80.

6. Pan RY, Ma J, Kong XX, Wang XF, Li SS, Qi XL, et al. Sodium rutin ameliorates Alzheimer's disease-like pathology by enhancing microglial amyloid- $\beta$ clearance. Sci Adv. 2019;5:eaau6328.

7. Ohnishi O. Search for the wild ancestor of buckwheat I. Description of new Fagopyrum (Polygonaceae) species and their distribution in China and the Himalayan hills. Fagopyrum. 1998;15:18-28.

8. Ohnishi O. Search for the wild ancestor of buckwheat III. The wild ancestor of cultivated common buckwheat, and of Tatary buckwheat. Econ Bot. 1998;52:123.

9. Ohnishi O, Konishi T. Cultivated and wild buckwheat species in eastern Tibet. Fagopyrum. 2001;18:3-8.

10. Yasui Y, Hirakawa H, Ueno M, Matsui K, Katsube-Tanaka T, Yang SJ, et al. Assembly of the draft genome of buckwheat and its application in identifying agronomically useful genes. DNA Res. 2016;23:215-24.

11. Zhang L, Li X, Ma B, Gao Q, Du H, Han Y, et al. The Tartary buckwheat genome provides insights into rutin biosynthesis and abiotic stress tolerance. Mol Plant. 2017;10:1224-37.

12. Varshney RK, Saxena RK, Upadhyaya HD, Khan AW, Yu Y, Kim C, et al. Whole-genome resequencing of 292 pigeonpea accessions identifies genomic regions associated with domestication and agronomic traits. Nat Genet. 2017:49:1082-8.

13. Varshney RK, Thudi M, Rookiwal M, He W, Upadhyaya HD, Yang W, et al. Resequencing of 429 chickpea accessions from 45 countries provides insights into genome diversity, domestication and agronomic traits. Nat Genet. 2019;51:857-64.

14. Fan W, Lu J, Pan C, Tan M, Lin Q, Liu W, et al. Sequencing of Chinese castor lines reveals genetic signatures of selection and yield-associated loci. Nat Commun. 2019;10:3418.

15. Zhou Z, Jiang Y, Wang Z, Gou Z, Lyu J, Li W, et al. Resequencing 302 wild and cultivated accessions identifies genes related to domestication and improvement in soybean. Nat Biotechnol. 2015;33:408-14.

16. Raizada A, Souframanien J. Transcriptome sequencing, de novo assembly, characterisation of wild accession of blackgram (Vigna mungo var. silvestris) as a rich resource for development of molecular markers and validation of SNPS by high resolution melting (HRM) analysis. BMC Plant Biol. 2019;19:358.

17. Bhardwaj A, Dhar YV, Asif MH, Bag SK. In silico identification of SNP diversity in cultivated and wild tomato species: insight from molecular simulations. Sci Rep. 2016;6:38715.

18. Batley J, Barker G, O'Sullivan H, Edwards KJ, Edwards D. Mining for single nucleotide polymorphisms and insertions/ deletions in maize expressed sequence tag data. Plant Physiol. 2003;132:84-91.

19. Yasui Y, Ohnishi O. Phylogenetic relationships among Fagopyrum species revealed by nucleotide sequence of the ITS region of the nuclear rRNA gene. Genes Genet Syst. 1998;73:201-10.

20. Honda Y, Mukasa Y, Suzuki T, Abe N. Stone buckwheat, genetic resources of buckwheat in Japan. In: "9th Int Symp Buckwheat", Prague. 2004; pp.185-9.

21. Suzuki T, Morishita T, Mukasa Y, Takegawa S, Yokota S, Ishiguro K, et al. Discovery and genetic analysis of non-bitter Tartary buckwheat (Fagopyrum tataricum Gaertn.) with trace-rutinosidase activity. Breeding Sci. 2014;64:339-43.

22. Sano M, Nakagawa M, Oishi A, Yasui $Y$, Katsube-Tanaka T. Diversification of 135 globulins, allergenic seed storage proteins, of common buckwheat. Food Chem. 2014;155:192-8.

23. Huang C, Zhang R, Gui J, Zhong Y, Li L. The receptor-like kinase AtVRLK1 regulates secondary cell wall thickening. Plant Physiol. 2018;177:671-83.

24. Feng $\mathrm{H}$, Chen Q, Feng J, Zhang J, Yang $\mathrm{X}$, Zuo J. Functional characterization of the Arabidopsis eukaryotic translation initiation factor 5A-2 that plays a crucial role in plant growth and development by regulating cell division, cell growth, and cell death. Plant Physiol. 2007;144:1531-45.

25. Zhou J, Li CL, Gao F, Luo XP, Zhao HX, Yao HP, et al. Characterization of three glucosyltransferase genes in Tartary buckwheat and their expression after cold stress. J Agric Food Chem. 2016;64:6930-8.

26. Sun Y, Li H, Huang J. Arabidopsis TT19 functions as a carrier to transport anthocyanin from the cytosol to tonoplasts. Mol Plant. 2012;5:387-400.

27. Liu Y, Jiang H, Zhao Y, Li X, Dai X, Zhuang J, et al. Three Camellia sinensis glutathione S-transferases are involved in the storage of anthocyanins, flavonols, and proanthocyanidins. Planta. 2019;250:1163-75. 
28. Zhao Y, Dong W, Zhu Y, Allan AC, Lin-Wang K, Xu C. PpGST1, an anthocyanin-related glutathione S-transferase gene, is essential for fruit coloration in peach. Plant Biotechnol J. 2019. https://doi.org/10.1111/pbi.13291.

29. Nesi N, Jond C, Debeaujon I, Caboche M, Lepiniec L. The Arabidopsis TT2 gene encodes an R2R3 MYB domain protein that acts as a key determinant for proanthocyanidin accumulation in developing seed. Plant Cell. 2001;13:2099-114.

30. Riechmann JL, Meyerowitz EM. The AP2/EREBP family of plant transcription factors. Bio Chem. 1998;379:633-46.

31. Kakei Y, Yamamoto M, Ishida Y, Yamazaki C, Sato A, Narukawa-Nara M, et al. Biochemical and chemical biology study of rice OsTAR1 revealed that tryptophan aminotransferase is involved in auxin biosynthesis: identification of a potent OsTAR1 inhibitor, pyruvamine2031. Plant Cell Physiol. 2017;58:598-606.

32. Shao $A$, Ma W, Zhao $X$, Hu M, He X, Teng $W$, et al. The auxin biosynthetic TRYPTOPHAN AMINOTRANSFERASE RELATED TaTAR2.1-3A increases grain yield of wheat. Plant Physiol. 2017;174:2274-88.

33. Suvorova G, Zhou M. Distribution of cultivated buckwheat resources in the world. In: Zhou M, Kreft I, Suvorova G, Tang Y, Woo SH, editors. Buckwheat germplasm in the world. London: Academic; 2018. p. 21-35.

34. Li C, Zhou A, Sang T. Rice domestication by reducing shattering. Science. 2006;311:1936-9.

35. Wang H, Nussbaum-Wagler T, Li B, Zhao Q, Vigouroux Y, Faller M, et al. The origin of the naked grains of maize. Nature. 2005;436:714-9.

36. Tan L, Li X, Liu F, Sun X, Li C, Zhu Z, et al. Control of a key transition from prostrate to erect growth in rice domestication. Nat Genet. 2008:40:1360-4.

37. Wang M, Li W, Fang C, Xu F, Liu Y, Wang Z, et al. Parallel selection on a dormancy gene during domestication of crops from multiple families. Nat Genet. 2018;50:1435-41.

38. Liu PL, Du L, Huang Y, Gao SM, Yu M. Origin and diversification of leucine-rich repeat receptor-like protein kinase (LRRRLK) genes in plants. BMC Evol Biol. 2017;17:47.

39. Li $\mathrm{H}$ et al. Aligning sequence reads, clone sequences and assembly contigs with BWA-MEM. arXiv:1303.3997v2, 2013.

40. Li H, Handsaker B, Wysoker A, Fennell T, Ruan J, Homer N, et al. The sequence alignment/map (SAM) format and SAMtools. Bioinformatics. 2009;25:2078-9.

41. Li H. A statistical framework for SNP calling, mutation discovery, association mapping and population genetical parameter estimation from sequencing data. Bioinformatics. 2011;27:2987-93.

42. McKenna A, Hanna M, Banks E, Sivachenko A, Cibulskis K, Kernytsky A, et al. The Genome Analysis Toolkit: a MapReduce framework for analyzing next-generation DNA sequencing data. Genome Res. 2010;20:1297-303.

43. Wang K, Li M, Hakonarson H. ANNOVAR: functional annotation of genetic variants from high-throughput sequencing data. Nucleic Acids Res. 2010;38:e164.

44. Xue AT, Hickerson MJ. The aggregate site frequency spectrum for comparative population genomic inference. Mol Ecol. 2015;24:6223-40.

45. Li H. TreeFam: a curated database of phylogenetic trees of animal gene families. Nucleic Acids Res. 2006;34:D572-80

46. Zheng X, Levine D, Shen J, Gogarten S, Laurie C, Weir B. A high-performance computing toolset for relatedness and principal component analysis of SNP data. Bioinformatics. 2012;28:3326-8.

47. Jombart T. adegenet: a R package for the multivariate analysis of genetic markers. Bioinformatics. 2008;24:1403-5.

48. Raj A, Stephens M, Pritchard JK. fastSTRUCTURE: variational inference of population structure in large SNP data sets. Genetics. 2014;197:573-89.

49. Chen H, Patterson N, Reich D. Population differentiation as a test for selective sweeps. Genome Res. 2010;20:393-402.

50. Ma Y, Ding X, Qanbari S, Weigend S, Zhang Q, Simianer H. Properties of different selection signature statistics and a new strategy for combining them. Heredity. 2015;115:426-36.

51. Pfeifer B, Wittelsbürger U, Ramos-Onsins SE, Lercher MJ. PopGenome: an efficient Swiss army knife for population genomic analyses in R. Mol Biol Evol. 2014;31(7):1929-36.

52. Kang HM, Sul JH, Service SK, Zaitlen NA, Kong S, Freimer NB, et al. Variance component model to account for sample structure in genome-wide association studies. Nat Genet. 2010;42:348-54.

53. Lippert C, Listgarten J, Liu Y, Kadie CM, Davidson Rl, Heckerman D. FaST linear mixed models for genome-wide association studies. Nat Methods. 2011;8:833-5.

54. Walker IH, Hsieh P, Riggs PD. Mutations in maltose-binding protein that alter affinity and solubility properties. Appl Microbiol Biotechnol. 2010;88:187-97.

55. Zhou M, Sun Z, Ding M, Logacheva MD, Kreft I, Wang Dm, et al. FtSAD2 and FtJAZ1 regulate activity of the FtMYB11 transcription repressor of the phenylpropanoid pathway in Fagopyrum tataricum. New Phytol 2017;216(3):814-828.

56. Zhang K, Logacheva MD, Meng Y, Hu J, Wan D, Li L, et al. Jasmonate-responsive MYB factors spatially repress rutin biosynthesis in Fagopyrum tataricum. J Exp Bot. 2018;69(8):1955-66.

57. Li J, Zhang K, Meng Y, Li Q, Ding M, Zhou M. FtMYB16 interacts with Ftimportin-a1 to regulate rutin biosynthesis in Tartary buckwheat. Plant Biotechnol J. 2019;17(8):1479-81.

58. Zhang K, He M, Fan Y, Zhao H, Gao B, Yang K, Li F, Tang Y, Gao Q, Lin T, Quinet M, Janovská D, Meglič V, Kwiatkowski J, Romanova O, Chrungoo N, Suzuki T, Luthar Z, Germ M, Woo SH, Georgiev MI, Zhou M. Resequencing of global Tartary buckwheat accessions reveals multiple domestication events and key loci associated with agronomic traits. Raw data sets for genetic diversity analysis and GWAS (PRJNA600676) https://www.ncbi.nIm.nih.gov/bioproject/PRJNA600676 /. Accessed 5 Aug 2020.

59. Zhang K, He M, Fan Y, Zhao H, Gao B, Yang K, Li F, Tang Y, Gao Q, Lin T, Quinet M, Janovská D, Meglič V, Kwiatkowski J, Romanova O, Chrungoo N, Suzuki T, Luthar Z, Germ M, Woo SH, Georgiev MI, Zhou M. Resequencing of global Tartary buckwheat accessions reveals multiple domestication events and key loci associated with agronomic traits. Github 2020, https://github.com/rahello/tartary_population. Accessed 5 Aug 2020.

60. Zhang K, He M, Fan Y, Zhao H, Gao B, Yang K, Li F, Tang Y, Gao Q, Lin T, Quinet M, Janovská D, Meglič V, Kwiatkowski J, Romanova O, Chrungoo N, Suzuki T, Luthar Z, Germ M, Woo SH, Georgiev MI, Zhou M. Resequencing of global Tartary buckwheat accessions reveals multiple domestication events and key loci associated with agronomic traits. Zenodo. 2020. https://doi.org/10.5281/zenodo.3972746.

\section{Publisher's Note}

Springer Nature remains neutral with regard to jurisdictional claims in published maps and institutional affiliations. 